\title{
The Multi Material Lightweight Vehicle (MMLV) Project
}




\title{
Other SAE books of interest:
}

\author{
Plastics Application Technology for Lightweight Automobiles \\ By Sudhakar Marur \\ (Product Code: R-415)
}

\section{Automotive Lightweighting Using Advanced High-Strength Steels \\ By Paul E. Geck \\ (Product Code: R-431)}

\author{
Design of Automotive Composites \\ By Charles Lu and Srikanth Pilla \\ (Product Code: PT-164)
}

\section{Lightweight Magnesium Technology 2001-2005 \\ By Thomas Ruden \\ (Product Code: PT-131)}

\author{
For more information or to order a book, contact: \\ SAE INTERNATIONAL \\ 400 Commonwealth Drive \\ Warrendale, PA 15096 \\ Phone: +1.877 .606 .7323 (U.S. and Canada only) \\ or +1.724 .776 .4970 (outside U.S. and Canada) \\ Fax: +1.724.776.0790 \\ Email: CustomerService@sae.org \\ Website: books.sae.org
}




\section{The Multi Material Lightweight Vehicle (MMLV) Project}

By Timothy Skszek, Jeff Conklin - Magna International Matthew Zaluzec, David Wagner - Ford Motor Company project co-funded by US Department of Energy

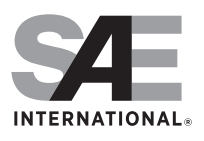

Warrendale, Pennsylvania, USA 


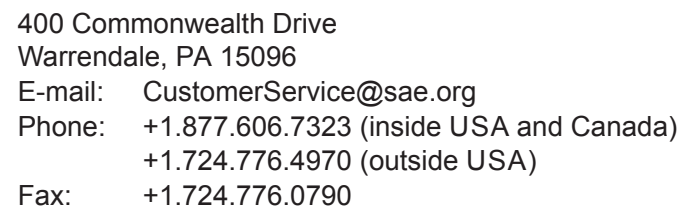

Copyright $@ 2015$ SAE International. All rights reserved.

No part of this publication may be reproduced, stored in a retrieval system, distributed, or transmitted, in any form or by any means without the prior written permission of SAE International. For permission and licensing requests, contact SAE Permissions, 400 Commonwealth Drive, Warrendale, PA 15096-0001 USA; e-mail: copyright@sae.org; phone: 724-772-4028; fax: 724-772-9765.

\section{Library of Congress Catalog Number 2015941402 SAE Order Number PT-170 http://dx.doi.org/10.4271/pt-170}

Information contained in this work has been obtained by SAE International from sources believed to be reliable. However, neither SAE International nor its authors guarantee the accuracy or completeness of any information published herein and neither SAE International nor its authors shall be responsible for any errors, omissions, or damages arising out of use of this information. This work is published with the understanding that SAE International and its authors are supplying information, but are not attempting to render engineering or other professional services. If such services are required, the assistance of an appropriate professional should be sought.
ISBN-Print 978-0-7680-8223-4
ISBN-PDF 978-0-7680-8224-1
ISBN-epub 978-0-7680-8226-5
ISBN-prc 978-0-7680-8225-8

To purchase bulk quantities, please contact

SAE Customer Service

e-mail: CustomerService@sae.org

phone: +1.877.606.7323 (inside USA and Canada)

+1.724 .776 .4970 (outside USA)

fax: +1.724 .776 .0790$

Visit the SAE Bookstore at

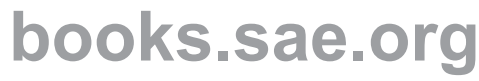




\section{Table of Contents}

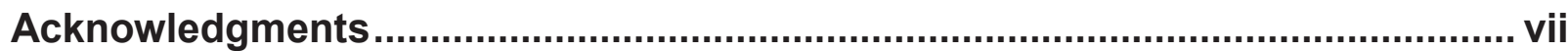

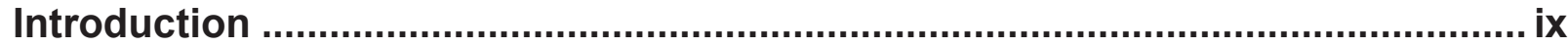

DOE Focuses on Developing Materials to Improve Vehicle Efficiency (2015-01-0405)

Schutte, Carol: Department of Energy Vehicles Technology

MMLV: Project Overview (2015-01-0407)

9

Timothy W. Skszek, Magna International; Matthew Zaluzec, Ford Motor Company; Jeff Conklin, Magna International; David Wagner, Ford Motor Company

BIW Design and CAE (2015-01-0408)

Jeff Conklin, Randy Beals, Zach Brown, Magna International

MMLV: Door Design and Component Testing (2015-01-0409)

Larry Plourde, Magna International; Michael Azzouz, Jeff Wallace, Ford Motor Co.; Mari Chellman, Magna International

MMLV: Lightweight Interiors Systems Design (2015-01-1236) 35

John Jaranson, Ford Motor Company; Meraj Ahmed, Eicher Engineering Solutions

MMLV: Chassis Design and Component Testing (2015-01-1237)

Xiaoming Chen, Ford Motor Company; Jeff L. Conklin, Robert M. Carpenter, Magna International; Jeff Wallace, Cynthia Flanigan, David A. Wagner, Vijitha Kiridena, Ford Motor Company; Stephane Betrancourt, Sogefi Group; Jason Logsdon, NHK Spring Group

MMLV: Aluminum Cylinder Block with Bulkhead Inserts and Aluminum Alloy Connecting Rod (2015-01-1238).

Cliff Maki, Kevin Byrd, Bryan McKeough, Ford Motor Co.; Robert G. Rentschler, Ford Casting Operations; Brian J. Nellenbach, Rick L. Williams, James M. Boileau, Ford Motor Co

MMLV: Carbon Fiber Composite Engine Parts (2015-01-1239)

Neal J. Corey, Mark Madin, Rick L. Williams, Ford Motor Co. 
MMLV: Automatic Transmission Lightweighting (2015-01-1240)........................ 71

James Kearns, Soon Park, Ford Motor Co.; John Sabo, Dusan Milacic, Magna International

MMLV: Corrosion Design and Testing (2015-01-0410) 83

Kevin Smith, Ying Zhang, Magna International

MMLV: Vehicle Durability Design, Simulation, and Testing (2015-01-1613)

Nikhil Bolar, Thomas Buchler, Magna International; Allen Li, Jeff Wallace, Ford Motor Co.

MMLV: Crash Safety Performance (2015-01-1614) 99

Yijung Chen, Derek Board, Omar Faruque, Cortney Stancato, James Cheng, Ford Motor Company; Nikhil Bolar, Sreevidhya Anandavally, Magna International

MMLV: NVH Sound Package Development and Full Vehicle Testing (2015-01-1615)

Yuksel Gur, Ford Motor Company; Jian Pan, Autoneum North America Inc; John Huber, Jeff Wallace, Ford Motor Company

MMLV: Life Cycle Assessment (2015-01-1616) 113

Lindita Bushi, Life Cycle Assessment Consulting; Timothy Skszek, Magna International; David Wagner, Ford Motor Company

MMLV Project Team 